\title{
De la conception néo-malthusienne du « discours de Dakar » de Sarkozy. Note de recherche
}

\author{
Aladji Madior Diop ${ }^{\mathrm{a}}$ and Richard Marcoux ${ }^{\mathrm{b}}$ \\ ${ }^{a}$ Groupe interuniversitaire d'études et de recherches sur les sociétés africaines (GIERSA), Pavillon \\ Charles-De Koninck, 1030, avenue des Sciences-Humaines, Université Laval, Québec QC Canada, \\ G1V 0A6, Courriel : aladji-madior.diop.1@ulaval.ca \\ b Département de sociologie, Faculté des sciences sociales, Pavillon Charles-De Koninck 1030, \\ avenue des Sciences-Humaines, Bureau 4491, Université Laval, Québec (Québec) G1V 0A6, \\ Courriel : richard.marcoux@soc.ulaval.ca
}

\section{Résumé}

En 2007, dans une allocution présentée au Sénégal, le Président français Nicolas Sarkozy déclarait: « la réalité de l'Afrique, c'est une démographie trop forte pour une croissance économique trop faible ". En une seule phrase, il résumait ainsi une idée centrale d'une doctrine de population, d'abord formulée par Malthus mais surtout relayée par les néomalthusiens depuis plus de deux siècles. Une analyse de contenu de ce discours révèle également d'autres éléments de convergence avec la doctrine néo-malthusienne. Ce qu'il est convenu de nommer maintenant «le discours de Dakar» a fait l'objet d'une masse importante de critiques différentes de la part de plusieurs chercheurs et intellectuels et que l'on peut retrouver dans quelques ouvrages clés publiés. A notre connaissance toutefois ce discours n'a jamais été examiné sous le prisme d'une doctrine de population ; une lacune que nous tenterons en partie de combler avec le présent article.

Keywords: Doctrine de population; néomalthusianisme; développement; croissance démographique; Afrique.

\begin{abstract}
In 2007, in a speech he made in Senegal, the French President Nicolas Sarkozy declared that "the reality of Africa is population growth which is too strong, and economic growth which is too weak". This single statement encapsulated the central idea of one theory of population, initially formulated by Malthus but mainly propagated by neo-Malthusians for over 200 years. An analysis of the speech also reveals other signs of adherence to neo-Malthusian theory. What is now known as the "Dakar Speech" has been subjected to a large body of different critiques by a variety of researchers and thinkers, which are available in certain key published works. However the speech seems never to have been examined through the lens of population theory; this article attempts to fill this gap.
\end{abstract}

Mots clés: Demographic theory; Neo-malthusian; Development; Population growth; Africa. 


\section{Introduction}

Au cours de l'histoire, les phénomènes de population ont toujours été la préoccupation de plusieurs grands penseurs des sciences humaines et sociales. Cela a fait dire à Gabriel Poulalion (1984:155) que "l'instinct de population est aussi vieux que l'homme sur terre ». Dès l'Antiquité nous renseigne Annie Vidal (1994), les hommes politiques grecs et romains se sont préoccupés de l'équilibre entre population et moyens de subsistance. Ce faisant, le souci d'assurer une meilleure qualité de vie à la population avait conduit Platon dans Les Lois à fixer le nombre idéal de citoyens de la cité à 5040 personnes (Charbit 2002). Dans le même ordre d'idées, Aristote, dans la Politique, esquissait quelques éléments d'une politique démographique pour contenir la population dans des limites acceptables. II a toutefois fallu attendre la fin du $15^{\mathrm{e}}$ siècle pour assister à l'émergence d'une réelle préoccupation des phénomènes de population dans les débats sociaux. Celleci, fondée sur des considérations purement économiques, sera l'apanage des mercantilistes et physiocrates tels que Jean Bodin, Richard Cantillon, François Quesnay, Antoine de Montchrestien, etc.

Cependant, c'est le nom du pasteur et économiste anglais, Thomas Robert Malthus, que l'histoire retiendra principalement pour l'étude des problèmes de population et tout particulièrement pour " cette discipline qu'est la démographie " (Marcoux 1998 : 173). En effet, dans son ouvrage intitulé Essai sur le principe de population, il sera le premier à jeter les bases d'une réelle démarche scientifique pour permettre de comprendre les phénomènes de population, et ce, en s'intéressant plus particulièrement aux liens entre population et moyens de subsistance. Ainsi, il aura une influence considérable sur bon nombre d'intellectuels alors que son nom sera même associé à un large mouvement doctrinaire, le néomalthusianisme, qui marquera les débats autour des enjeux en matière de population depuis plus de 200 ans (Minois 20I I).

Le $20^{\mathrm{e}}$ siècle, qui a vu la réflexion démographique se mondialiser (Poulalion 1984), aura également été marqué par ces débats houleux entre tenants et adversaires de la perspective néomalthusienne. Certes, une certaine accalmie est observée, notamment depuis la dernière conférence internationale qui s'est tenue au Caire en 1994 et au cours de laquelle la plupart des États s'accordaient à reconnaître la nécessité d'une meilleure maîtrise de l'accroissement démographique. Cette accalmie s'explique bien évidemment par le fort ralentissement de la croissance démographique observé dans plusieurs régions du monde. La chute rapide de la fécondité, observée en Asie et en Amérique latine depuis les années 1960 et 1970, semble avoir rassuré les plus inquiètes des milieux néomalthusiens alors que le processus de vieillissement des populations du Nord, durant la même période, aura même conduit à un changement complet de paradigme pour cette région du monde avec la mise en place de mesures visant plutôt à favoriser une remontée de la fécondité.

En réalité, seule l'Afrique fait actuellement bande à part avec les nombreux pays de ce continent qui maintiennent des niveaux de fécondité de plus de 5 ou 6 enfants par femme et des niveaux de croissance démographique de plus de 2,5\% par an (Marcoux et Konaté 2010). Peut-on alors s'attendre à ce que le discours néo-malthusien se concentre sur le continent africain? C'est du moins ce que semble avoir confirmé Nicolas Sarkozy dans son fameux discours de Dakar lorsqu'il affirme: "la réalité de l'Afrique, c'est une démographie trop forte pour une croissance économique trop faible ". Quel est le sens de ces propos relativement aux liens entre population et développement? Qu'est-ce qui peut, dans les éléments du discours prononcé à Dakar en 2007 par le Président français, conduire à un rapprochement avec les perspectives néomalthusiennes?

Pour répondre à ces questions, nous proposons, dans un premier temps, une présentation très succincte des éléments fondamentaux qui caractérisent la conception néomalthusienne liant la population à l'économie. Dans un second temps, nous tenterons de dégager ce qui nous apparaît être les éléments de convergence entre les propos tenus par le président français et la doctrine néomalthusienne.

Ce qu'il est convenu de nommer maintenant «le discours de Dakar» a fait l'objet d'une masse importante de critiques différentes de la part de plus d'une cinquantaine de chercheurs et d'intellectuels et que l'on peut retrouver dans différents ouvrages clés $(\mathrm{Ba}$ Konaré 2008; Gassama et al. 2008 ; Chrétien 2008 ; Mbem 2008; Traoré 2008). A notre connaissance toutefois, ce discours et le débat qui l'entoure n'ont jamais été examinés sous le prisme des doctrines de population; une lacune que nous tenterons en partie de combler avec le présent article.

\section{Les éléments fondamentaux de la concep- tion néomalthusienne des liens entre popu- lation et économie}

Apparu dès le $19^{\mathrm{e}}$ siècle, le néomalthusianisme s'est développé d'abord en Angleterre avant de se propa- 
ger dans d'autres pays et notamment en France qui nous intéresse ici plus particulièrement (Lux 1998). C'est au lendemain de la Seconde Guerre mondiale que cette doctrine prendra une dimension planétaire en intégrant notamment les préoccupations environnementales et du coup les milieux écologistes et certaines agences internationales. Mais un mot d'abord sur Malthus.

\section{L'héritage de Malthus}

Pasteur anglican, Thomas Robert Malthus était profondément touché par la misère sociale grandissante et surtout par l'inutilité des efforts fournis par les pouvoirs publics britanniques pour l'éradiquer. Ainsi, il s'élève contre les diverses « lois des pauvres " qu'il considère comme des remèdes aggravant le mal. Dans son ouvrage Essai sur le principe de population, dont la première édition paraît en 1798 et qui constitue une des références majeures de l'économie classique du $19^{\mathrm{e}}$ siècle, Malthus construit son raisonnement à partir de deux postulats de base, à savoir :

- «Premièrement, que la nourriture est nécessaire à l'existence de l'homme;

- Deuxièmement, que la passion réciproque entre les sexes est une nécessité, et restera à peu près ce qu'elle est à présent " (Malthus 1980 [1798] : 24).

Néanmoins, souligne Malthus, il y a problème dans la mesure où le pouvoir multiplicateur de la population est infiniment plus grand que le pouvoir qu'a la terre de produire la subsistance de l'homme. En effet propose-t-il, alors que la population s'accroît en progression géométrique, les moyens de subsistance n'augmentent qu'en progression arithmétique, ce qui provoque indubitablement un déséquilibre qui serait à l'origine d'innombrables problèmes. Malthus avance que l'histoire montre que le retour à l'équilibre entre la population et les moyens de subsistance n'est rendu possible que par l'apparition de freins répressifs ou régulateurs tels que les famines, les disettes ou les guerres. Afin d'éviter d'en arriver là, Malthus propose de favoriser un obstacle préventif, ce qu'il nomme la contrainte morale, qui consiste principalement au recul de l'âge au mariage et bien sûr à l'abstinence de relations sexuelles hors mariage. Foncièrement opposé à ce qu'il nomme "le vice ", Malthus s'est empressé d'affirmer: "Je repousserai toujours, comme étant immoral (...) tout moyen artificiel et hors des lois de la nature que l'on voudrait employer pour contenir la population » (Malthus, cité par Ronsin $1980: 31$ ).
En dépit de l'abondance et de la virulence des critiques adressées à l'encontre de la personne de Malthus et de son œuvre, force est de reconnaître que nombreux sont les intellectuels et scientifiques qui s'inspireront de ses thèses en les vivifiant et en les élargissant à d'autres domaines de la vie économique et sociale.

\section{Genèse et évolution du néomalthusianisme}

Déjà du vivant de Malthus, quelques religieux, comme Joseph de Maistre, prônaient la généralisation de sa doctrine. De plus, certains ténors de l'économie classique n'ont jamais caché leur admiration et leur adoption des thèses de Malthus. À cet effet, Stuart Mill défendait l'idéal stationnaire au nom de ce que l'on nomme la qualité de la vie, tandis que Ricardo faisait du principe de population de Malthus une pièce maîtresse de la théorie de la répartition. Darwin, pour sa part, soutenait s'inspirer de Malthus lorsqu'il écrivit son ouvrage Origines des espèces (Vidal 1994).

Cependant, l'année 1822 sera marquée par un élément majeur dans le débat suscité par l'œuvre de Malthus. La publication du livre Illustration et preuves du principe de population par Francis Place ouvrit une nouvelle voie dans la pensée malthusienne. Prônant et menant campagne pour l'adoption de procédés anticonceptionnels et d'hygiène intime pour lutter contre la surpopulation, Place mit fin au " moral restreint " défendu par Malthus ouvrant ainsi la voie à ce qu'il est convenu d'appeler le néomalthusianisme.

Cette doctrine qu'est le néomalthusianisme se développera très vite durant la deuxième moitié du $19^{\mathrm{e}}$ siècle avec ses principaux tenants: Georges et Charles Robert Drysdale en Angleterre et Paul Robin en France. Ils ne lésineront pas sur les moyens : journaux, livres, conférences et tracts leur serviront de moyens privilégiés de propagande. Ils mettront sur pied des organisations susceptibles de leur procurer une certaine légitimité juridique et un meilleur cadre de sensibilisation tout en assurant la diffusion de leurs journaux et périodiques (Ronsin 1980; Drouard 1992).

Comme le rappelle André Lux (1998), trois postulats de base constituent l'épine dorsale de la doctrine néomalthusienne. D'abord, les néomalthusiens soutiennent que le principe de population est une loi biologique qui sous-tend toutes les sciences sociales. Ensuite, ils voient en la surpopulation la principale et unique responsable de tous les maux qui affectent l'individu et la société. Enfin, ils estiment que le néomalthusianisme est l'unique remède à tous ces 
maux. Par conséquent, la doctrine néomalthusienne apparaît pour ses adeptes comme le passage obligé, la condition sine qua non du progrès et du bien-être. Enfin, les néomalthusiens considèrent la surfécondité comme un comportement criminel et le contrôle de l'immigration, une obligation pour l'État (Lux 1998).

Ces postulats admis, les néomalthusiens adoptent toutes les thèses de Malthus en rejetant toutefois et sans ménagement le " moral restreint ». Ils sont convaincus qu'il n'y a qu'une seule solution aux deux problèmes auxquels l'humanité est confrontée, à savoir, le partage équitable des biens matériels et les joies de l'amour et, cette solution n'est rien d'autre que la diffusion à grande échelle des moyens artificiels pour éviter la fécondité et ainsi ralentir la croissance démographique.

Alors que les deux guerres mondiales et les pertes humaines qui les ont accompagnées conduiront à une marginalisation des mouvements néomalthusiens pendant la première moitié du $20^{\circ}$ siècle, la rapide croissance démographique observée dans les pays de l'hémisphère Sud et largement médiatisée dès le début des années 1960 (Valette 1990), servira de prétexte et de tremplin à un important regain de popularité des mouvements néomalthusiens. Ceux-ci définiront cette croissance démographique du Sud comme une bombe à retardement (Ehrlich 1969). Avec une population composée à plus de $60 \%$ de jeunes, éduquer, soigner, nourrir, loger et assurer un emploi pour tous devient alors un défi lancé aux États (Vidal 1994). Ces défis qui se posent sont à l'origine de plusieurs chantiers de recherches-actions sur la fécondité et la planification familiale qui vont permettre de mieux connaître les tendances en matière de natalité, mais surtout, d'identifier les sociétés et pays où les comportements des populations favorisent une forte croissance démographique. Certains de ces chantiers bénéficieront de l'appui des médias et de quelques leaders d'opinion et hommes politiques, mais aussi de l'éclairage scientifique de certains démographes et de certaines institutions, américaines plus particulièrement (Greenhalgh 1996).

De par leurs actions, les mouvements néomalthusiens auront ainsi une influence considérable sur les décideurs politiques, mais aussi sur certaines institutions des Nations Unies et sur d'autres institutions de développement (De Koninck 1998). Au nom de l'impératif du développement, certaines institutions internationales seront invitées à lier leur aide à l'adoption et à la mise en œuvre de politiques de limitation des naissances par les États bénéficiaires (Ehrlich 1972; Valette 1990; McNamara 1992).
Au terme de cette analyse succincte, nous constatons que les éléments fondamentaux de la conception néomalthusienne liant population et développement ont beaucoup contribué à la catégorisation de certaines sociétés qui seraient insuffisamment avancées dans leur transition démographique; le maintien d'une fécondité élevée représentant ainsi un frein à leur développement, voire même une menace pour l'ensemble de la planète (Ehrlich et Ehrlich 1990).

\section{Convergence entre le discours de Sarkozy et la doctrine néomalthusienne}

C'est le 26 juillet 2007 dans la capitale sénégalaise, quelques mois après avoir remporté l'élection présidentielle française, que Nicolas Sarkozy a prononcé un discours rendu célèbre notamment par la vague de réactions qu'il a suscitées dans le cercle des intellectuels, africains et français plus particulièrement. Empruntant des propos fréquemment utilisés par les tenants de l'afro-pessimisme, ce discours propose une vision sombre du continent africain. Examinons d'abord le contexte dans lequel le discours de Dakar a été prononcé.

Durant les décennies 1980-2000, la quasi-totalité des pays africains ont mis en pratique des programmes d'ajustement structurel (PAS) sous le contrôle des institutions de Bretton Woods. Ces PAS, qui avaient pour objectif de permettre à ces pays de faire face aux problèmes économiques structurels aigus auxquels ils étaient et sont toujours confrontés, ont eu des effets sociaux désastreux, notamment une recrudescence des inégalités et de la pauvreté. Partout, les problèmes sociaux se sont multipliés à cause des restrictions budgétaires et de l'augmentation du coût global de la vie.

Les jeunes, qui représentent plus de la moitié de la population dans ces pays, ont été les principales victimes de cette crise. Sans ressource et livrés à eux-mêmes, certains d'entre eux sont attirés par les sirènes de l'immigration. Ainsi, depuis 2006, des milliers d'entre eux en provenance de l'Afrique subsaharienne, dont de nombreux Sénégalais, ont bravé la mer et ses intempéries à bord d'embarcations de fortune pour aller en Europe clandestinement avec le slogan "Barça ou Barçax " (Barcelone ou la mort).

Dès la campagne électorale présidentielle, Nicolas Sarkozy avait tenu des propos qui pouvaient lui permettre de faire des gains auprès des électeurs charmés par le discours du Front national, parti d'extrême droite ouvertement hostile à l'immigration. II faut déjà se rappeler qu'à titre de 
ministre de l'Intérieur, en 2006, sous la présidence de Jacques Chirac, il élabore le Projet de loi Sarkozy sur «l'immigration choisie» dans lequel il est notamment proposé de faire subir des tests ADN aux futurs candidats au regroupement familial, dont plusieurs demandes proviennent du continent africain.

C'est donc dans ce contexte et fort de cette réputation que Nicolas Sarkozy a effectué en 2007 sa première visite officielle sur le continent africain en tant que Président de la République française. Le point d'orgue de cette visite en terre africaine a été le discours prononcé à l'Université Cheikh Anta Diop de Dakar devant un parterre d'intellectuels, d'hommes politiques et d'étudiants. $\mathrm{Ce}$ fut l'occasion de s'adresser à la jeunesse sénégalaise et, au-delà de celle-ci, à toute la jeunesse africaine sur les questions majeures de l'avenir de l'Afrique subsaharienne et de ses rapports avec la France.

\section{Les axes du discours de Dakar}

Le discours de Nicolas Sarkozy, rédigé par son conseiller Henri Guaino, est essentiellement axé sur trois espaces temporels que nous reprendrons ici succinctement, à savoir le passé, le présent et l'avenir de l'Afrique. Le premier point a été, pour lui, l'occasion de revenir sur quelques moments sombres de l'histoire du continent africain, le Président Sarkozy rappelant les souffrances endurées par les Africains durant la traite négrière qui a vidé l'Afrique de ses hommes et de ses femmes parmi les plus valides. À ce titre, affirme Nicolas Sarkozy, la traite négrière a été un crime contre l'homme, un crime contre l'humanité tout entière. Dans la même veine, il avancera que la conquête coloniale et la colonisation sont aussi atroces que l'esclavage. Néanmoins, à ses yeux, la colonisation apparaît comme un mal nécessaire en ce sens qu'elle a permis de construire en Afrique l'essentiel des infrastructures : routes, ponts, écoles, hôpitaux, etc.

S'adressant largement à son public à la première personne du singulier, loin de se disculper ou de se repentir des fautes commises par ses aïeuls, il dira que l'Afrique a sa part de responsabilité dans son malheur, car la colonisation ne serait pas responsable de toutes les difficultés actuelles de l'Afrique, avance-t-il. Ces difficultés, qui ont pour noms guerres sanglantes, génocides, dictatures, fanatisme de tout genre, gaspillage et pollution, sont aujourd'hui le lot quotidien des Africains et sont surtout dues au fait que l'homme africain serait mal entré dans l'histoire. Sans histoire, vivant au rythme des saisons, où l'instinct joue un plus grand rôle que la raison, le paysan africain vit dans l'imaginaire d'un monde presque exclusivement rural, où il n'y a pas de place ni pour l'aventure humaine ni pour l'idée de progrès. Poursuivant son analyse des problèmes actuels de l'Afrique, Sarkozy affirme que l'une des réalités de l'Afrique, c'est une démographie trop forte pour une croissance économique trop faible. Ce déséquilibre a pour conséquence trop de famine et trop de misère, entraînant la rareté qui à son tour, suscite la violence. Aussi, soutient-il, la réalité de l'Afrique, c'est encore et surtout un développement au ralenti et une agriculture peu productive, c'est l'insuffisance des infrastructures routières, des écoles et des hôpitaux.

Abordant le thème de l'immigration, une autre réalité de l'Afrique, Nicolas Sarkozy dit avoir bien compris l'envie de partir qu'éprouvent un si grand nombre de jeunes Africains confrontés aux difficultés de l'Afrique. Cependant, croit-il, la jeunesse africaine n'est pas poussée à partir pour fuir la misère, mais plutôt parce que, comme toute jeunesse, elle veut conquérir le monde.

Par ailleurs, le Président français affirme que l'Afrique ne veut pas de la charité, ne veut pas d'aide, ni de passe-droit. Ce dont l'Afrique a besoin soutient Nicolas Sarkozy, c'est lutter contre la famine, la mortalité infantile et la pollution. Pour y parvenir, il serait nécessaire pour les Africains de chercher l'autosuffisance alimentaire, de développer les cultures vivrières, de produire pour se nourrir. L'Afrique a aussi besoin de paix, de démocratie, d'unité, de justice sociale, d'une application de l'État de droit, mais également d'une coopération et d'un partenariat entre des nations égales en droits et en devoirs et c'est à cela que la France veut convier les Africains. Pour ce faire, le Président français déclare que la France mènera une politique de partenariat avec l'Afrique qui sera basée sur le codéveloppement, c'est-à-dire le développement partagé. Ce partenariat sera axé sur une politique d'immigration négociée pour permettre à la jeunesse africaine d'être accueillie en France et en Europe avec dignité et avec respect. Enfin, souligne-t-il, l'Eurafrique sera le cadre privilégié pour exprimer ce nouveau type de partenariat.

Ce discours suscitera de nombreuses controverses et fera couler beaucoup d'encre. Notre intention ici n'est pas de revenir sur tous les termes du débat. Dans les lignes qui vont suivre, nous nous efforcerons simplement d'identifier les éléments du discours qui convergent avec de nombreux éléments de la doctrine néomalthusienne, objet de notre analyse. 


\section{Éléments de convergence}

Il serait hasardeux à partir de ce discours de qualifier le Président Nicolas Sarkozy de néomalthusien. Comme pour plusieurs autres acteurs et auteurs qui se prononcent sur les débats concernant le développement, les enjeux de population demeurent marginaux dans sa pensée et dans ses actions. Néanmoins, les propos tenus dans son discours et son action politique peuvent-ils laisser entrevoir une certaine affinité avec le néomalthusianisme ou une source d'inspiration privilégiée dans la caricature qu'il propose de l'Afrique et des Africains? Ses propos sur l'immobilisme du paysan africain, l'immigration, l'absence de croissance économique pour cause d'une démographie galopante sont autant d'éléments qui convergent avec la perspective néomalthusienne.

Le premier point de convergence observé a d'abord trait à la forme des discours. Tout comme les néomalthusiens de la fin du $19^{\mathrm{e}}$ siècle et du début du $20^{\circ}$ siècle (Drouard 1992 ; Lux 1998), mais également certains " amis de l'Afrique " (Mbokolo 2008: 3), Nicolas Sarkozy emploie un style direct ponctué de répétitions pour convaincre son auditoire. À la manière de ces derniers, arrogance, sarcasme et provocation accompagnent ses propos, mais aussi «mépris, méconnaissance et racisme » (Bâ Konaré 2008 : 21). À la suite de Victor Hugo (cité par Mbokolo 2008 : I2) qui affirmait en 1879 que " l'Afrique n'a pas d'histoire » et de René Dumont, qui lançait en 1966 que l'Afrique est mal partie, Nicolas Sarkozy soutient que " l'homme africain est mal entré dans l'histoire et que l'Afrique et les Africains ont une part de responsabilité dans leur propre malheur»' (Sarkozy 2007). Notons au passage que Sarkozy partage avec les néomalthusiens l'idée selon laquelle les couches défavorisées, tout comme les nations attardées et affamées, sont victimes de leur manque de rationalité et sont ainsi « coupables par leur imprévoyance et leur incontinence d'avoir violé le droit naturel à l'existence "(Meillassoux 1991 : 18-19).

Un autre signe caractéristique des convergences entre les propos de Sarkozy et la perspective néomalthusienne est perceptible sur la description qu'il fait « du paysan africain condamné à vivre dans une éternelle routine au rythme des saisons et dont l'idéal de vie est d'être en harmonie avec la nature. Dans cet imaginaire où tout recommence toujours, il n'y a pas de place ni pour l'aventure humaine ni pour l'idée de progrès ". Cette caricature du paysan africain, plus particulièrement et de l'homme africain en général et de son supposé manque de rationalité, est présente tout au long du discours et reprend des idées longtemps soutenues et relayées par Malthus à propos des pauvres et encore davantage par les tenants du néomalthusianisme. Effectivement, déjà en I798, Malthus mettait l'accent dans son Essai sur

«(...) l'insouciance et le manque d'économie que l'on peut observer chez les pauvres qui semblent toujours vivre au jour le jour. Ainsi leurs besoins les plus immédiats occupent-ils toute leur attention de même qu'ils pensent rarement à l'avenir. Même quand ils ont l'occasion d'épargner, ils ne le font presque jamais, mais tout ce qui dépasse leurs besoins du moment va, en général au cabaret » (Malthus 1798 [1980]: 52).

Aussi, cette attitude de l'ancien Président français s'inscrit dans le paradigme "qui trouve dans l'opposition entre tradition et modernité l'explication du "mal africain" " (Bâ Konaré 2008: 95). Aux yeux des tenants de ce paradigme, I'homme africain semble frapper par une incapacité de changer le cours des choses pour s'ouvrir à la modernité (Bâ Konaré 2008). Du reste, cette vision des Africains, qui date de la colonisation, a toujours été utilisée pour expliquer ce qui est entendu comme étant " immobilisme ". Effectivement, très fréquemment, les administrateurs coloniaux dans leurs rapports décrivaient la situation alimentaire des colonies en mettant en exergue " le fatalisme qui caractériserait les populations africaines face aux calamités naturelles, leur incapacité de prévenir et de gérer les crises alimentaires » (Gado $2008: 83$ ).

Par ailleurs, en lançant sa désormais célèbre phrase : « la réalité de l'Afrique, c'est une démographie trop forte pour une croissance économique trop faible ", Sarkozy résume en une seule formule tout le principe de population formulé par Malthus et largement relayé par les néomalthusiens depuis plus de deux siècles. Toute la littérature néomalthusienne est axée sur cette liaison entre population et croissance économique. Les tenants de cette doctrine n'ont jamais cessé de tenter de démontrer que la forte poussée démographique, quand elle n'est pas accompagnée d'une forte croissance économique, entraîne inéluctablement une pression sur les subsistances.

Du reste, le décalage entre population et croissance économique sera considéré comme un signe probant de sous-développement. Pour les néomalthusiens, les raisons de cet écart sont à rechercher dans un trop fort taux de natalité et un relativement faible taux de mortalité. Ils soulignent que le déca- 
lage entre une démographie galopante et une économie faiblement dynamique dans les pays sousdéveloppés, particulièrement africains, est dû au fait que ces pays n'ont pas encore pu être transformés par la modernisation (Greenhalgh 1996). Selon cette approche, les mesures sanitaires ou sociales importées dans ces pays auraient permis l'expansion effrénée de la population, tandis qu'aucune action d'envergure n'a permis le changement des techniques, des structures économiques, des institutions et des relations internationales (Dumont et Rosier 1966). Ehrlich (1969) de renchérir en soutenant que chaque année la production alimentaire dans ces pays prend un peu plus de retard par rapport à l'accroissement irrésistible de la population.

Pour les néomalthusiens, plus la population augmente, plus les terres cultivables deviennent rares et les infrastructures insuffisantes et inadaptées. Ainsi, comme le dit Nicolas Sarkozy, l'agriculture ne produit pas assez et cela se répercute inéluctablement sur la nutrition. D'où, la famine, la mortalité et la pollution. "La réalité de l'Afrique, c'est encore trop de famine, trop de misère. La réalité de l'Afrique, c'est la rareté qui suscite la violence» nous dit Nicolas Sarkozy; des thèmes constamment présents et au cœur des discours néomalthusiens et les tenants de l'afro-pessimisme.

\section{Conclusion}

Le rapprochement de quelques éléments du discours de Dakar avec les composantes de la doctrine néomalthusienne révèle assurément certains points de convergence. Aussi bien pour les néomalthusiens que pour Nicolas Sarkozy, les réalités auxquelles l'Afrique est confrontée et qui ont pour noms famines, disettes et guerres, sont dues à la fatalité et à l'imprévoyance des populations africaines (Gado 2008) et qui conduisent à «une démographie trop forte pour une croissance économique trop faible».

Tout comme les néomalthusiens, et rejoignant ici les afro-pessimistes, Nicolas Sarkozy occulte le rôle de la colonisation. John O. Igué (2008), rappelle au contraire que l'« immobilisme » dont Nicolas Sarkozy et ses «inspirateurs» font état, trouve ses origines dans la colonisation. En effet, les sociétés traditionnelles africaines avaient mis en place des systèmes de gestion et de prévention des crises de subsistance très efficaces permettant aux paysans de faire face aux aléas climatiques et aux périodes de soudure (Gado 2008). Malheureusement, les transformations forcées des activités agricoles initiées par les colons avaient pour objectif de mettre en place un cadre qui devait permettre de fournir les matières premières dont avait besoin la métropole. Dès lors, estime John O. Igué (2008), l'introduction des cultures de rente en Afrique a engendré beaucoup plus de problèmes qu'elles n'en ont résolus. Elle est à l'origine de nouvelles techniques culturales peu adaptées et à forte intensité de main d'œuvre, des migrations forcées des travailleurs des régions peu propices aux cultures de rente vers les zones plus favorables, entraînant ainsi une destruction des sociétés rurales. Autant d'éléments qui peuvent, en partie, expliquer le maintien de comportements favorisant une natalité élevée.

Par ailleurs, au-delà du discours prononcé à Dakar, les déclarations et l'action politique de Nicolas Sarkozy semblent en avoir fait la personne idéale pour mettre en application certains idéaux des néomalthusiens en matière d'immigration. Comme le souligne Amartya Sen (1995), la "peur d'être englouti» par l'immigration du Sud est une image qui accompagne depuis plusieurs années la rhétorique concernant la «bombe démographique» qui menacerait l'Occident. Même si il faut reconnaître que l'immigration n'est pas un thème dominant du néomalthusianisme, comme nous le rappelle André Lux (1998), le contrôle de l'immigration par l'État est très vite présenté comme une nécessité chez les néomalthusiens. Par exemple, le néomalthusien James Blanchard soutenait à la fin du $19^{\mathrm{e}}$ siècle que : " la France est littéralement envahie et submergée par les races criminellement prolifiques qui franchissent ses frontières, les Belges, les Allemands, les Italiens. (Ces derniers) se répandent partout dans le pays, arrachant le pain de la bouche des Français, les concurrençant à la baisse sur leur propre marché du travail " (Blanchard dans la revue The Mathusian de décembre 1884, cité dans Lux $1998: 213$ ).

À sa suite, Lacombe, une figure marquante du néomalthusianisme français, craignait l'immigration d'étrangers en France au début du $20^{\text {ième }}$ siècle et appela les autorités à travailler afin d'empêcher ce "trop-plein d'étrangers envahissant la France» (Lacombe dans la revue Génération consciente de novembre 1908 et cité par Lux $1998: 213$ ).

Quelques mois avant son investiture comme candidat, le futur président déclarait devant ses militants: "On en a plus qu'assez d'avoir en permanence le sentiment de s'excuser d'être Français. On ne peut pas changer ses lois, ses coutumes parce qu'elles ne plaisent pas à une infime minorité. Si certains n'aiment pas la France, qu'ils ne se gênent pas pour la quitter» (Nicolas Sarkozy cité dans Le Monde, 
édition du 23 avril 2006). Peut-on croire que ceux dont il est question ici ne sont plus belges, allemands et italiens, mais plutôt originaires d'Afrique? On peut alors comprendre que les propositions concernant «une politique d'immigration négociée», qu'on retrouve dans le discours de Dakar, n'aient pas suscité beaucoup d'enthousiasme.

\section{Contributions des auteurs}

Les deux auteurs ont tous les deux contribué à l'obtention des données nécessaires à la rédaction du texte et se sont partagé les tâches de recensions des écrits et d'analyse des informations. De plus, la rédaction finale du texte a pu se faire à travers plusieurs échanges et lectures croisées. Aladji Madior Diop a pour sa part présenté sous forme de communication orale, une première version provisoire de cet article dans un colloque à l'Université Laval en 2009.

\section{Bibliographie}

Ba Konaré., A. (sous la direction de) 2008. Petit précis de remise à niveau sur l'histoire africaine à l'usage du président Sarkozy. Paris: La Découverte.

Charbit, Y. 2002. « La Cité platonicienne : histoire et utopie ", Population, vol. 57, no 2 : 23I-260

Chrétien, Jean-Pierre (sous la direction). 2008. L'Afrique de Sarkozy. Un déni d'histoire, Karthala, Paris, $204 \mathrm{p}$.

De Koninck, M. 1998. "Discours féminisme et néomalthusianisme : les effets pervers d'une mésalliance», Cahiers québécois de démographie, vol. 27, no 2 : 253-266

Dumont, R. et Rosier, B. 1966. Nous allons à la famine. Paris : Éditions du Seuil.

Drouard, A. 1992. "Aux origines de l'eugénisme en France: le néomalthusianisme (1896-1914) », Population, Vol. 47, No. 2 : 435-459.

Ehrlich, P.1969. La bombe P, Paris : Fayard/Les Amis de la terre.

Ehrlich, P. R. 1972. "World population: A battle lost?" Population Crisis. An Interdisciplinary Perspective, Glenview : $12-17$

Ehrlich, P. R. and A.H. Ehrlich. 1990. The Population Explosion, Simon and Schuster, New York.

Gado, B. A. 2008. "La gestion et la prévention des crises de subsistance dans les sociétés précoloniales du Sahel : mythe ou réalité?» Petit précis de remise à niveau sur l'histoire africaine à l'usage du président Sarkozy. Paris : La Découverte : 83-94
Gassama, M., M. Diagne, D. Diop et K. Lamko. 2008. L'Afrique répond à Sarkozy : Contre le discours de Dakar, Philippe Rey, 478 p.

Greenhalgh, S. 1996. «The Social Construction of Population Science: An Intellectual, Institutional, and Political History of Twentieth-Century Demography». Comparative Studies of Society and History, 38(I) : 26-66

Igué, J. O. 2008. «Le rôle de la colonisation dans l'« immobilisme " des sociétés africaines». Petit précis de remise à niveau sur l'histoire africaine à l'usage du président Sarkozy. Paris: La Découverte : $215-226$

Lux, A. 1998. "Les ligues néo-malthusiennes françaises de 1896 à 1940 : idéologie de droite sous un manteau ouvriériste». Cahier québécois de démographie, 27 (2) : 199-219

Malthus, T.R. 1980 [1798]. Essai sur le principe de population en tant qu'il influe sur le progrès de la société avec des remarques sur les théories de Mr, Godwin et de $M$. Condorcet et d'autres (première édition), traduction par Éric Vilquin. Paris : Institut National d'Études Démographiques (INED).

Marcoux, R. 1998. «Thomas R. Malthus et son Essai sur le principe de population, 200 ans plus tard». Cahier québécois de démographie, 27(2) : I80-188

Marcoux, Richard et Mamadou Kani Konaté (20II). "Africa and the Francophonie of Tomorrow: Attempt to Measure the Population of the Francophonie from Now to 2060", African Population Studies, volume 25, no 2, p. 215-225

Mbem, A. J. 2008. Nicolas Sarkozy à Dakar. Débats et enjeux autour d'un discours. Paris : L'Harmattan.

Mbokolo, É. 2008. «Préface», Petit précis de remise à niveau sur l'histoire africaine à l'usage du président Sarkozy. Sous la direction de A. Ba-Konaré, Paris : La Découverte.

McNamara, R.N. 1992. «The Population Explosion», The Futurist, novembre-décembre 1992: 9-13

Meillassoux, C. 1991. «Les leçons de Malthus: le contrôle démographique par la faim». Les spectres de Malthus. Paris : EDI/ORSTOM/CEPED, 15-32

Minois, G. 20II. Le poids du nombre. L'obsession du surpeuplement dans l'histoire. Paris : Perrin.

Piché, V. et Marcoux, R. 1995a. «Les enjeux fondamentaux de la Conférence du Caire sur la population». Les Échos de l'ADQ, 22(3) : 6-8

Poulalion, G. 1984. La science de la population. Paris : Litec.

Ronsin, F. 1980. La grève des ventres. Propagande néomalthusienne et baisse de la natalité française ( $X I X^{\mathrm{e}}-X X^{\mathrm{e}}$ siècles). Paris : Aubier-Montaigne. 
Sarkozy N. 2007. Allocution prononcée à l'Université de Dakar, le 26 juillet 2007, [En ligne]: http://www.afrik.com/articlel2199.html (page consultée le 30 août 20I I).

Sen, A. 1995. "Menace démographique et misère économique. II n'y a pas de bombe démographique ». Esprit, no 216, pp. II8-147
Traoré, A. 2008. L'Afrique humiliée. Paris: Fayard.Valette, R. 1990. La population mondiale: des situations géographiques et des problèmes différents sinon opposés. Éthique et démographie. Lyon: PROFAC, 13-26

Vidal, A. 1994. La pensée démographique. Doctrines, théories et politiques de population. Grenoble: Presses Universitaire de Grenoble.

\section{NOTES}

' Les extraits cités du discours de Nicolas Sarkozy, prononcé à l'Université Cheikh Anta DIOP de Dakar le 26 juillet 2007 sont repris intégralement entre guillemets suivi de l'indication (Sarkozy 2007). L'intégralité du discours se retrouve sur le web notamment: http://www.afrik.com/article12199.html (page consultée le 11 avril 2014). On retrouve également l'intégralité du discours sur WIKIPEDIA. 